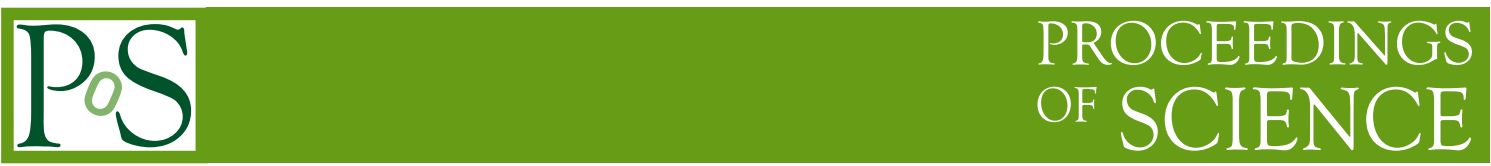

\title{
Online Track Reconstruction at Hadron Colliders
}

\author{
Silvia Amerio* \\ INFN Padova \\ E-mail: silvia.amerio@pd.infn.it
}

\begin{abstract}
The online reconstruction of tracks is a fundamental element of trigger systems in High Energy Physics experiments, especially in the presence of very crowded events at high instantaneous luminosity. In this paper we will review the main characteristics of online tracking processors at hadron colliders. Moving from the past to the future, and towards increasing instantaneous luminosity, we will first show how $\mathrm{H} 1$ experiment at Hera faced the challenges of online track reconstruction implementing pattern matching and track linking algorithms on CAMs and FPGAs in the Fast Track Trigger (FTT). The pattern recognition technique is also at the basis of the Silicon Vertex Trigger (SVT) at the CDF experiment at Tevatron: coupled to a very fast fitting phase, SVT allows to trigger on displaced tracks, thus greatly increasing the efficiency for the hadronic B decay modes. A recent upgrade of the SVT track fitter, the Gigafitter, can perform more than 1 fit/ns and further improves the CDF online trigger capabilities at high luminosity. At SLHC, where luminosities will be three orders of magnitude greater than Tevatron, online tracking will be much more challenging: we will describe CMS future plans for a Level-1 track trigger and the Fast TracK (FTK) processor at the ATLAS experiment, based on the SVT architecture and designed to provide high quality tracks reconstructed in time for a Level-2 trigger decision.
\end{abstract}

35th International Conference of High Energy Physics - ICHEP2010,

July 22-28, 2010

Paris France

\footnotetext{
*Speaker.

$\dagger$ On behalf of H1, CDF, ATLAS and CMS Collaborations
} 


\section{Introduction}

Real time event reconstruction plays a fundamental role in High Energy Physics experiments. Reducing the rate of data to be saved on tape from millions to hundreds of events per second is critical. In order to increase the purity of the collected samples, rate reduction has to be coupled with a first selection of the most interesting events. Online selections based on tracks are more effective than those based on calorimetric objects, especially in the case of very crowded events: a calorimetric trigger tower integrates the energy from all the particles in the event, without distinction between the hard scattering and the pile-up events, while online tracking allows to distinguish the particles based on their $z$ coordinate and transverse momentum $p_{T}$.

An online tracking processor has to identify tracks in a few $\mu s$ at Level-1 (L1) and only tens of $\mu s$ at Level-2 (L2), dealing with data from thousands of readout channels: the combinatorial and computational task is extremely demanding. A possible approach to this problem, common to all the devices described in this paper, is the pattern matching technique: a track in a detector is a set of hits (pattern), one per each layer traversed by the charged particle; if all possible patterns generated by particles in the detector can be stored in a memory, a track candidate is found when the set of real hits matches one of the stored patterns. A pattern matching algorithm can be implemented on a parallel architecture, thus it is particularly suitable to online tracking where very high speed is needed.

\section{The Fast Track Trigger at $\mathrm{H1}$}

The Fast Track Trigger (FTT) [1] was in operation at H1 detector at HERA collider from 2004 to 2007. It was built after an increase of a factor five in instantaneous luminosity and allowed to preserve the capability to select very rare decays such as $D^{*} \rightarrow K \pi \pi$. In the FTT a pattern matching algorithm was implemented with Content Addressable Memories (CAMs) in high density Altera FPGAs. It was used both at L1 and, with increased resolution, at L2 to identify tracks using a subset of the data from the $\mathrm{H} 1$ central drift chamber. Besides the pattern matching technique, a key element of FTT is the usage of the same flexible and multipurpose board (MultiFunctional Processing Board [2]) in different stages of the pipeline: to merge data streams, link track segments both at L1 and L2, fit tracks and take the trigger decision at L2. The usage of the same board made the system much easier to develop and maintain. With FTT high performance was achieved not only for triggering $D^{*}$ mesons but also for triggering electron and muons from $b$ quark decay [3].

\section{The experience of the Silicon Vertex Tracker at CDF}

The Silicon Vertex Tracker [4] at CDF, since 2002 allows to reconstruct tracks with offline-like resolution (for example $35 \mu \mathrm{m}$ on the impact parameter) and in time for a $\mathrm{L} 2$ decision $(\sim 20 \mu \mathrm{s})$. Three key features allow SVT to carry out the track reconstruction in such a short time: firstly SVT has a highly parallel architecture, being divided into 12 engines, each working independently on the data from one of the 12 sectors of the silicon detector. Secondly, SVT implements a pattern matching algorithm by means of custom VLSI Associative Memory chips [5]. Finally, track fitting at high resolution is performed only inside the matched patterns and is thus reduced to a simple 
scalar product, that can be processed very quickly in a FPGA. The SVT system, as FTT, is based on the same multipurpose board, the PULSAR [6], widely used in the CDF trigger system as it can interface with any CDF data format by means of simple mezzanine cards. The PULSAR is at the basis of the most recent upgrade of SVT (2010): the track fitting system, composed by 16 boards, has been substituted by a single board, the Gigafitter (GF) [7], which can perform more than 1 fit/ns at the working frequency of $120 \mathrm{MHz}$. It is based on a very powerful FPGA, the Xilinx Virtex 5, which is particularly suitable to perform scalar products, being provided with as many as 640 DSPs. The GF allows to recover track reconstruction efficiency in regions of the silicon detector not covered by the old track fitting system and to extend the SVT acceptance on track transverse momentum and impact parameter.

\section{Online tracking at SLHC}

At LHC the online reconstruction of tracks is much more demanding than at Hera or Tevatron. At instantaneous luminosities of the order of $10^{34} \mathrm{~cm}^{-2} \mathrm{~s}^{-1}$ tens of multiple interactions per event are expected. This number will increase to hundreds at SLHC, where the instantaneous luminosity will be one order of magnitude higher. Currently not ATLAS nor CMS can trigger on tracks at L1 and only lately at L2, but both experiments are studying online tracking processors at low trigger level for the SLHC phase.

\subsection{Triggering on tracks at L1: possible solutions at CMS.}

The data volume is one of the main challenges to face when developing an online tracking processor to be used in time for a L1 decision: as an example, at the SLHC instantaneous luminosity, a data rate of $\sim 10-20 \mathrm{~Gb} \mathrm{~cm}^{-2} \mathrm{~s}^{-1}$ will be needed to read out a single silicon detector layer at a distance of $10 \mathrm{~cm}$. A fast online data reduction is thus essential. A possible solution is keeping for the $\mathrm{L} 1$ decision only the tracks with $p_{T}>1 \mathrm{GeV}$, and removing the low momentum ones which would be anyhow rejected by the trigger but account for the vast majority of the tracks produced in a high luminosity event. At CMS two possible methods [8] based on this idea are under study. Stacked tracking [9] aims at correlating hits between two closely separated sensors using a simple pattern matching algorithm. Hit pairs falling outside a search window are removed as they likely belong to low momentum (great curvature) tracks. With multiple sensor pairs is then possible to match the surviving hit pairs into raw tracks. A method based on cluster width discrimination [10] is also under study: in a four layer detector, low $p_{T}$ tracks are rejected based on the greater width (greater curvature) of the clusters of hits in each layer. A correlation between the surviving clusters from three out of four layers can then be performed using CAM or FPGAs implementing a pattern matching technique á la SVT.

\subsection{Online tracks at L2: the FTK at ATLAS}

The Fast TracK (FTK) [11] is a processor under development at ATLAS to reconstruct offlinelike tracks in time for a L2 decision. It is based on the SVT architecture, adapted to the ATLAS environment, where working conditions are much more demanding: FTK has to sustain a L1 frequency of $100 \mathrm{KHz}$ instead of the $30 \mathrm{KHz}$ of SVT; the silicon detector at CDF is composed by five layers of sensors, while FTK has to process the data from 11 layers; as a consequence the pattern 
bank size is much greater and more than $800 \mathrm{M}$ of patterns are necessary to reconstruct tracks with a good efficiency, to be compared with the $6 \mathrm{M}$ needed by SVT. A new Associative Memory chip is under development to meet the pattern needs of FTK and track fitting will be performed on a small mezzanine card directly mounted on the back of the Associative Memory board. Simulations have shown [12] that the availability of offline-like tracks at L2 will allow to implement single muon triggers with a flat signal efficiency as a function of the number of multiple interactions, thanks to isolation cuts based on tracks rather than on energy deposits in the calorimeters; moreover, online $b$ and $\tau$ jet reconstruction will be possible with efficiencies comparable to the offline.

\section{Conclusions}

Online tracking at low trigger level is very important to collect high purity samples at high instantaneous luminosity. $\mathrm{H} 1$ experiment at Hera and CDF at Tevatron have built fast and efficient online tracking systems, implementing a pattern matching technique by means of standard CAMs in FPGAs or custom Associative Memory chips. The flexibility and modularity of the systems are key elements of their success, as well as the usage, when possible, of single multipurpose boards. $\mathrm{H} 1$ and CDF experience will be very useful for the future experiments: currently both CMS and ATLAS are developing online tracking systems at low trigger level for the SLHC phase, where the reconstruction of tracks will be fundamental to preserve the capability of selecting interesting physics signals in the presence of hundreds of multiple interactions per event.

\section{References}

[1] A. Baird, et al., A Fast Resolution Track Trigger for the H1 Experiment, arxiv:hep-ex/0104010v1.

[2] D. Meer, et al., A Multifunctional Processing Board for the Fast Track Trigger of the H1 Experiment, arxiv:hep-ex/0107010v1.

[3] A. W. Jung, et al., First results from the Third Level of the H1 Fast Track Trigger, Proceedings of 15th IEEE-NPSS Real Time Conference (2007).

[4] A. Bardi et al., The CDF Online Silicon Vertex Tracker, r NIM A485 178-182 (200).

[5] A. Annovi et al., A VLSI Processor for Fast Track Finding Based on Content Addressable Memories, IEEE Transactions on Nuclear Science, Volume 53, Issue 4, Part 2, Aug. 2006 Page(s):2428 - 2433.

[6] J. Adelman et al., The Silicon Vertex Trigger Upgrade at CDF, NIM A572, 361 (2005).

[7] S. Amerio et al., The Gigafitter: Performance at CDF and Perspectives for Future Applications, 2010 J.Phys.: Conf.Ser. 219022001.

[8] G. Hall, Design of a Trigger Module for the CMS Tracker at SLHC, Proceedings of TWEPP-2009.

[9] J. Jones et al., A Pixel Detector for Level-1 Triggering at SLHC, arxiv:physics/0510228.

[10] F. Palla, Proposal for a First Level Trigger using Pixel Detector for CMS at S-LHC, 2007 JINST 2 $\mathrm{P} 02002$.

[11] A. Annovi et al., Associative Memory Design for the Fast TracK Processor (FTK) at Atlas, Proceedings of 17th IEEE-NPSS Real Time Conference (2010).

[12] A. Andreani et al., The FastTrack Real Time Processor and Its Impact on Muon Isolation, Tau and B-jet Online Selections at Atlas, Proceedings of 17th IEEE-NPSS Real Time Conference (2010). 